\title{
The Use of a Ward's History in Training Psychiatric Child Care Workers
}

\author{
Jeffrey L. Binder \\ Blue Ridge Community Mental Health Center, Charlottesville, Virginia \\ Jeffrey Urist \\ University of Michigan Medical Center, Ann Arbor
}

John G. Looney

Timberlawn Psychiatric Hospital, Dallas, Texas

\begin{abstract}
The importance of the contribution of historical-cultural factors to an inpatient treatment setting's contemporaneous psycho-social climate has been given little attention in published discussions of ward staff problems and training. This report illustrates some ways in which such historical-cultural forces, embodied in social myths and entrenched traditions, operate. Examples are provided from a ward of disturbed adolescents, where young child care workers struggled as a group with the daily stresses of providing therapeutic milieu support to children ambivalently seeking and resisting external controls for their feelings and impulses. The process of incorporating the analysis of historical-cultural factors in an inservice training program is described.
\end{abstract}

\section{Introduction}

Child care workers in a residential setting for adolescents need to be attuned to the way they function as individuals and as a group, because their patients use them as identification models. As adolescents are striving for identity formation and consolidation, they are acutely sensitive to the attitudes and values of adults who care for them. They eagerly try on for size the traits, attributes, and ideas of admired adults. Alternately, adolescents desire to know how these adults feel about things, so that they can defiantly take an opposing stance. By doing so, adolescents fortify their sense of individuality. If the adults who care for them are acting out role conflict, adolescent inpatients will not have therapeutically

Requests for reprints should be sent to Jeffrey L. Binder, Outpatient Service, Blue Ridge Community Mental Health Center, 1602 Gordon Avenue, Charlottesville, Virginia 22903. 
constructive models for identification. An important way to ensure that child care staff feel confident, function effectively, and provide adequate modeling is to help them understand and exercise control over the social forces prevailing in the milieu.

Most of the literature on the training of psychiatric care staff concurs that the staff are important contributors to the psychosocial climate of the milieu. Different staff training procedures, however, put varying degrees of emphasis on the contributing factors. One view emphasizes intrapsychic factors that motivate people to work on a psychiatric ward for children or adolescents, and attention is directed toward the interpersonal dynamics played out in the dyadic relationship between worker and patient (Alt, 1953; Bettelheim \& Wright, 1955, Maier, Hilgeman, Shuyart, \& Loomis, 1955; Rosen, 1963; Bettelheim, 1966). A second approach to training focuses on interpersonal relationships, emphasizing ways in which the network of interrelationships among staff affect patients they treat. This view stresses openness and clarity of communication among child care workers, doctors, and patients. The interpersonal dynamics of the entire ward are considered, and interest is primarily on contemporaneous vicissitudes in these dynamics (Szurek, 1947; Stanton \& Schwartz, 1954; Montalvo \& Pavlin, 1966; Pierce, Trickett, \& Moos, 1972; Krohn, Miller \& Looney, 1974). A third view, while noting that treatment is affected by emotional relationships among all staff, suggests that emotional climate is largely a function of the organizational properties of the ward, properties such as power constellations, communiction channels, and role definitions. In this view, it is important to understand a ward's interpersonal dynamics, but only as far as is necessary to diagnose maladaptations in the formal organizational structure of the ward (Stanton \& Schwartz, 1954; Henry, 1954, 1957).

It is our contention that these varied approaches to training child care workers have directed insufficient attention to an important perspective. While they all, in varying degrees, have acknowledged the importance of social forces influencing the treatment milieu, these forces tend to be viewed cross-sectionally, neglecting the longitudinal or historical perspective. Personal historical factors are considered when interpersonal processess are analyzed in terms of transference-countertransference binds engineered by patients. When, however, events are viewed from the perspective of a ward's social organization, there is a tendency to analyze social forces influencing child care workers and patients as though they germinated spontaneously from the activity of the 
present, unconnected to people and events from the ward's past. In training child care staff, more emphasis should be placed on historical-cultural aspects of a ward's social organization. The social factors influencing a milieu in the present are directly linked to and influenced by the milieu's cultural past.

The social organization of a psychiatric ward embodies a culture: a set of implicitly shared perspectives for interpreting events, and a set of shared modes of coping with them (Parkes, 1965). This culture also provides implicitly shared channels for expressing impulses and feelings stimulated by life on the ward. Events in the milieu can become part of cultural history and consequently can have repercussions over time, influencing the meanings child care staff attach to subsequent events and their affective reactions to them.

Historical factors affecting a ward's culture can come into sharpest focus during periods of stress. Such periods provide fertile ground for the germination of social myths, which arise less from historic truth than from a need to give order to painfully conflictual events (Slater, 1966). The forms taken by these myths are heavily influenced by the body of cultural beliefs accumulated over time, which serve to organize and give meaning to the contemporary realities of a particular milieu.

The importance of cultural-historical forces in the life of an adolescent psychiatric ward will be illustrated by examining several such myths which arose out of the progression of day-today stresses within this mini-culture. The importance of cultural myths in shaping staff attitudes and behavior can be seen in various areas of the milieu. The following aspects of the social organization of the ward are representive of major conflictual areas around which cultural myths tend to crystallize: (a) myths regarding libidinal and aggressive expression on the ward; (b) myths regarding the meaning and handling of racial differences on the ward; and (c) myths regarding ward leadership and autonomy. Before going into these particular areas of the functioning of the ward, however, a general introduction to the setting of this study is in order.

\section{The Setting and Method}

The events described below took place in the inpatient treatment unit of the adolescent psychiatry service of a university teaching hospital. 
Patients on the ward range in age from 13 to 18 years. The unit census includes 18 youngsters, 8 male and 10 female. The patients represent a broad spectrum of psychopathology ranging from severely neurotic problems to chronic schizophrenia. The psychoanalytically oriented treatment approach was an integration of milieu programs, counseling groups, and individual psychotherapy. A youngster's length of stay may range anywhere from a brief evaluation of four to six weeks to two years of long-term treatment. The child care staff is composed of 8 registered nurses and 17 child care workers. (In this report both nurses and non-nurses are included under the term "child care staff" because their duties overlap greatly. Individual and group therapists for patients and their parents are not included in the term "child care staff," although they, of course, take part in the policy setting for the handling of individual patients.) Though occupational and recreational therapists and school teachers comprise a significant ancillary milieu treatment force which clearly shared in the culture and the conflicts of the ward, our focus here is on the child care staff itself.

The staff tends to range in age from early twenties to early thirties. Many of the nurses tend to be recent nursing school graduates. Most of the non-nurse care workers are recent college graduates, a large percentage of whom eventually pursue graduate training in psychology, social work, or medicine. The ratio of male to female staff roughly parallels the ratio of male to female patients. The average length of tenure for ward staff is $11 / 2$ years. Turnover of staff generally tends to be evenly distributed over time. Although there may be occasional disruptive periods of relatively higher turnover, this was not felt to be a significant factor in the events described below.

Data for this analysis were generated from training groups set up for the child care staff, as well as from daily observations made on the ward. There were two groups, composed of members from the day and evening shifts, respectively. They each met once a week for $1 \frac{1}{2}$ hours, and were led by two of the authors. As they went on, leadership roles were also assumed by some child care workers. Meetings were open-ended. Discussion topics tended to focus on specific clinical problems and general ward issues raised by the ward staff. The general aim of the group meetings was to augment and integrate the clinical discussion of such issues with an examination on the part of the staff of their emotional responses to the particular situation. The manner, however, in which a few enduring, collectively shared fantasies repeatedly influenced their view of ward life attested to the need to include in the training an understanding of the way historical-cultural forces were coloring intrastaff relationships and attitudes toward patient care. The events reviewed below cover a range of approximately three years. The groups began meeting approximately one year from this time frame, so that the group process described here is drawn from roughly two years of regular group meetings. ${ }^{1}$

IA complete description of the staff training groups can be found in J. Binder, "A Model for Small-Group Training of Psychiatric Ward Staff," Psychiatry, 1976, 39, 364-375. 


\section{Results}

\section{Myths Regarding Libidinal and Aggressive Expression}

The emotional-biological pressures brought to bear on the organization of the personality during adolescence are great. It goes without saying that one of the major functions of the therapeutic milieu involves offering external supports to the youngster's ego capacities, particularly in the area of regulating feelings, impulses, and behavior. It is often precisely here that the adolescent's support system, provided by family members and others in his environment, has broken down. In this situation, our patients, who have been unable to manage these internal pressures, turn to ward staff as external ego and superego surrogates. The staff's demonstrated attitudes toward their own feelings and impulses, as well as those of their patients, ultimately serve as models for internalization. The crucial role of ward staff in this process stems directly from the fact that adolescents internalize models of behavior not through being targets of sermonizing but through experiencing how staff help them in their daily living and coping with emotional struggles.

As members of a group whose raison d'etre and daily existence involve dealing with the continual pressure to help patients manage and regulate feelings and behavior, ward staff come collectively to reflect an implicitly agreed-upon set of values, attitudes, affects, and coping mechanisms, which transcends the individual personality of each member. It is this culturally shared set of attitudes, rooted in the staff's common group experience, that to a large extent determines the effectiveness of the milieu in meeting the needs of disturbed adolescents. This is the setting in which group myths can be observed growing out of the staff's collective attempts to make their greater maturity available to adolescents who demand structural support for coping with their sexual and aggressive impulses. An example of this occurred during a training group session. The group began with a somewhat sarcastic discussion of a new "romance" on the ward.

Rick and Barb, both aged 15, had been holding hands while watching television. One of the male staff reported how he had directed "a raised eyebrow" at Rick, who immediately let go of Barb's hand. In discussing the handling of this type of situation, the group briefly alluded in a joking way to the discomfort they felt in intervening when "kids were making out" but quickly concluded with much resolve that "this type of thing had to be stopped quickly." It 
was suggested that the therapists of these two youngsters be notified of the staff's concern, with the strong recommendation that Rick and Barb be restricted from spending any time together. This suggestion was received by some of the more experienced staff with a great deal of pessimism. They expressed the belief that therapists never take this kind of acting out seriously until "one of the kids get pregnant." Furthermore, they argued, it was impossible to treat patients on this ward once they became emotionally involved with each other.

These conclusions were traced back to a similar situation that had arisen on the ward nearly two years earlier. At that time the therapists of two 15-year-olds had permissively allowed their patients' acting out to escalate to the point where they were constantly clinging to each other and were "rumored" to be having intercourse. Yet the therapists refused to follow up on the staff's expressions of concern. Whereas there clearly had been a breakdown in the staff-therapist alliance at that time, it was striking how that episode generated a myth which for two years served to insulate the staff from their own conflictual feelings about sexuality on the ward. Equally striking was the eagerness and ease with which new staff accepted as gospel the mythologized version of what older staff predicted about the milieu.

It is frequently the case that when ward staff began to examine their attitudes and behavior around a particular milieu problem, they discover that they have become part of a scenario orchestrated by the internal conflicts of the patients involved. In Barb's case it was discovered that in fact there had been a myth in this youngster's family that paralleled the myth on the ward. Barb's mother, who had gotten pregnant with this child at age 15, maintained and acted on a belief that unless her adolescent daughter were firmly prevented from meeting with boys, she would inevitably become pregnant, as the mother had. Barb was accompanied by the mother to all activities outside of school. Not surprisingly, the girl became pregnant at age 15, had an abortion, became depressed, and was eventually hospitalized.

The importance of the staff's becoming aware of its own mythmaking is obvious in such examples, where the individual treatment is clearly enhanced once these dramatic unconscious conflictual themes are fed back into the treatment rather than being blindly reenacted on the ward. Beyond consideration of isolated treatment cases, however, examining these myths has a far more pervasive effect on the entire milieu. It was clear, for example, that the staff's responsiveness to all patients around the issue of sexuality was enhanced by their recognizing together their own discomfort in addressing in an empathic way the patients' conflicted behavior. This work was not possible, however, until the staff as a group had recognized and traced to its source the myth 
that had served to isolate them from their own discomfort and from the needs of their patients.

\section{Myths Regarding the Meaning and Handling of Racial Differences}

There are a number of social problems that exist in the larger society which under closer scrutiny in the more circumscribed setting of the ward become more comprehensible in dynamic terms. Without going into the varied points of view regarding the psychology of prejudice, we suggest that in the particular situation of an adolescent ward, racial differences tend to be one of many vehicles by which adolescents attempt to split their environment into conflicting factions so as to coincide with their own internal, unsynthesized images of themselves and of others. Disturbed youngsters in particular tend toward these all-or-none arbitrary divisions, so that people are seen as either good or bad, weak or strong, loving or cruel. These divided, unintegrated images serve to protect the youngster from anxiety stemming from conflictual wishes, feelings, and attitudes. The attempt at organizing people into dichotomous categories represents another way in which the deficient ego of the youngster gives over its attempt to cope with conflict to the external environment. Here again, the more mature staff is looked to by the patient to synthesize and manage that with which his own ego cannot adequately come to terms.

Rossman and Miller (1973) discuss the way certain kinds of internal stress can precipitate acute and focused episodes of racial prejudice on an adolescent ward. The present focus, however, is on how the staff accepted the patient's use of splitting along racial lines, because they were apprehensive about dealing with their own racial tensions.

The staff meeting began with a discussion of Yvette, a 16-year-old newly admitted girl who was black, acutely psychotic, and creating a great deal of furor on the ward. Several of the staff who were white commented matter-of-factly on their difficulty in approaching her, since she seemed to be directing a great deal of unmitigated rage at any and all white staff who tried to approach her. There was general agreement that because Yvette was apparently "so afraid of white staff" it would be preferable for black staff to take responsibility for her until she demonstrated less fear of whites. At this point, one of the group leaders asked how people felt about this arrangement for working with Yvette. The feeling of most of the group was that considering the appalling disorder of her family history, much of Yvette's difficulty had to do with the fact that as a black youngster, she was disadvantaged, oppressed, and a victim of a system which 
black staff understood far more intimately than did white staff. The assumption that finally emerged was that Yvette's problem had to do more with her blackness than with her psychosis.

The implicit assumption that underlay this surprising formulation emerged only after the group dealt with a series of events that had taken place on the ward 11/2 years previously. This earlier episode involved Ryan, a psychotic black 16-year-old boy. Two staff members, one white and one black, who had worked closely with Ryan had gotten into serious disagreements over his treatment. Jim, the black staff member, had felt that Ruth, his white colleague, was passively jealous of his rapport with Ryan. Ruth had felt excluded and saw Jim as overprotective and engulfing their patient. Although there was little overt confronting of this issue at the time, the staff as a group were aware of the conflict. Much of the strong feeling generated by this problem became encapsulated in the form of a myth that white staff could not work effectively with black patients. While there are no doubt some realistic advantages in the richness of shared experience that may exist between patient and staff or patient and therapist of similar backgrounds, the defensive aspects of this myth far overshadowed any such realistic considerations. This particularly myth was primarily fueled by the staff's needs to rationalize and cover over tensions that tended to arise when racial factors entered into their work. In Yvette's case, only after these tensions were confronted could the staff, both black and white, began to treat her psychosis.

\section{Myths Regarding Ward Leadership and Staff Autonomy}

It is around the conflictual question of autonomy, perhaps more than within any other area of adolescent concern, that one sees clearly the interaction between he needs of patients and the social organization of the staff. Krohn, Miller, and Looney (1974) have discussed the manner in which adolescent conflicts over autonomy manage to tap related conflicts in those individuals who treat and care for them. The ability of staff to come to grips with these conflictual feelings is understood to be a major factor in their gaining the capacity to function autonomously, thereby providing their patients with developmentally appropriate role models. As such, the attempt to address these conflictual feelings around autonomy became a major focus in the staff group meetings described here.

A child care worker's professional identity, based upon a sense of clinical competence and relative autonomy in performance of tasks, is difficult to achieve when the worker is unsure of his ability to function autonomously, or if he even wants to. The members of a ward staff are often led by someone who, by formal status and perhaps also by age and force of personality, is seen as a strong 
leader. Such leaders can come to embody a professionally confining but secure set of role expectations with which a child care staff can grow comfortable. A staff's ambivalence around gaining autonomy may be circumvented by attributing to the leader alone the capacity for making critical decisions and initiating action; this occurs at the expense of each staff member's sense of personal growth. When ambivalence about autonomy is handled in this way, a social myth can arise which expresses the belief that the staff can function effectively only with a firm, directive leader; a more democratic form of leadership, with greater individual initiative and responsibility, would lead to chaos.

In fact, this very type of strong leader, Tom, had held formal responsibility for the daily milieu activities on the ward for several years. When he left to take another position, the child care workers, even though individually they were creative and energetic, floundered in their new autonomy.

Originally, Tom had been a regular member of the ward staff, but his unusually energetic and assertive manner, as well as his special talent for working with adolescents, gradually led to his assuming informal leadership over the rest of the staff. He also was older than most of the child care workers, which facilitated his acquisition of a leadership role. With Tom taking charge of milieu activities, the ward ran more smoothly than it had when each staff member was more responsible for his own activities. While each child care worker's autonomy was being eroded, both ward staff and therapists appreciated the increased surface tranquility and the way this man would intervene in crises, thereby relieving staff and therapists of that responsibility. Consequently, the informal leadership role that had evolved was transformed by the institution into a formal role. Furthermore, over time the origin of Tom's role was overshadowed by the growing belief that he and his role were present because they were indispensible.

This myth was maintained by an unconscious desire prevalent among the staff to avoid recognizing their capacities for autonomous functioning and, thus, to avoid accepting that aspect of their milieu roles that required them to be parenting adults for the adolescent patients. Most of the child care staff were young adults in their early twenties. Many were using this job as a holding action while they plotted their vocational futures. In many ways, then, their presence on the ward was expressive of a psychological moratorium (Erikson, 1959). When working under a paternal leader, the staff could see themselves as carrying out orders of an elder without feeling ultimately responsible for the 
content of those orders. This situation conveniently meshed with their personal need to avoid commitment to a particular set of ideals and values. It allowed them to maintain a collective fantasy that their relationship to their adolescent charges was one of older siblings to younger siblings. When faced with more responsibility for the standards and values prescribed for their patients, these young child care workers were intensely ambivalent about enforcing many of the milieu's rules, because these rules were experienced as "adult" values of the therapists and consequently threatened to foreclose prematurely their own search for values and identities.

In one training session there was much discussion about the difficulty in maintaining interest in confronting patients about such apparently mundane issues as mesy rooms, appropriate manners at meals, and proper dress. It was acknowledged that when dealing with patients in clear emotional crisis, the workers felt like helpful clinicians, but when hassling patients about the details of social living, they felt uncomfortably like parents-often bitchy, fuddyduddy parents. Accompanied by much kidding and laughing, several workers in turn described how after a shift they would let their hair down and do such things as become incredibly sloppy at home and talk in a very gross manner to roommates. Several of them related how one time after work they went out to a nearby airport, where they ate ice cream and chased each other around the terminal like a bunch of kids.

These highly competent child care workers needed to engage in this occasional regressive behavior in order to reassure themselves that they were not irreversibly trapped in what they felt to be premature adulthood. The alternative route was to re-identify temporarily with their own past adolescences and childhoods. This struggle sometimes blinded the child care workers to the significant therapeutic need for confronting disturbed youngsters with their laxness in taking care of themselves and their environment. It was difficult for them to see therapeutic parenting behavior, which can be tremendously beneficial to disturbed adolescents, as part of their role. They felt, in essence, that senior staff, by delegating this job to the child care staff after the old leader had left, had abdicated their "parental" responsibility.

This young child care staff displayed enthusiasm, creativity, and impressive clinical sensitivity. They did not shirk responsibility for facing the patients' often unsettling emotional storms. But taking a responsible role in the milieu's operation burdened them with the unwanted yoke of adulthood. A part of each of them 
yearned for a return to the comfortable apprentice-like role each had known before. This wish was indirectly evidenced in one training group session around a discussion of the Community Projects Program to be staffed jointly with the patients (this was a program of various helping projects with the poor and aged, which provided the patients with the experience of giving to others instead of always being placed in a position of receiving):

In this session, the discussion centered around several workers' reluctance to get involved in various projects. It soon became clear that most care workers did not want to participate in projects when they felt any inadequacy, uncertainty, or anxiety. The fantasy surfaced that staff-patient participation in the Community Projects should, in essence, involve the patient being apprenticed to a wise staff guildmaster. The care worker should be confident, without anxiety, and highly skilled at the task, while the patient could be anxious but eager to learn from the staff member. The idea that two individuals could work together, both possibly feeling uncertain about how best to go about the task but determined to work and learn together, was not easily considered. It seemed that the child care workers' fantasies about staff-patient relationships in a community project mirrored their own wishful memories of working under their departed ward leader.

It was striking how even relatively new staff who had never known Tom tended to share in the overall group assumptions about the handling of responsibility on the ward. Exploring the staff's feelings regarding the history of ward leadership became a focus for examining their discomfort with the parental expectations of their job. The effectiveness of the group's work in this area was nicely reflected in the groups themselves, where there tended to be a gradual but clear increase in staff initiative and leadership.

\section{Discussion and Conclusions}

The staff training groups have several goals, which have been enumerated previously. They also serve the essential function of providing an opportuniy for ward staff to preserve and keep alive the history of their milieu. By taking time to examine current milieu problems and issues with the explicit mental set that the ward's history may shed light on current experiences, older staff are encouraged to retrieve memories of earlier events and people. These older staff in turn pass on their memories to newer staff, who eventually become the keepers of the ward's history for the next generation of staff. Through this process a ward staff consciously 
maintains a history of its milieu, which it can use to understand and control the myths and traditions that are maintained through implicit consent and that are inevitable under stressful conditions.

In general, when organizing an in-service training program, it is helpful first to elucidate the treatment philosophy of the ward. In particular, the general goals of patient care around which the therapeutic milieu is organized should be clearly stated. Once this general framework is articulated, the interpersonal structure of the milieu can be scrutinized in terms of whether it fosters or impedes the milieu's therapeutic goals. Then, for a thorough understanding of present aspects of a ward's organization, particular attention should be given to their historical-cultural antecedents. There are a number of aspects of the interpersonal structure of the milieu that can be examined with an eye to their historical development.

When looking at the leadership structure, for instance, one must determine whether the degree of freedom given to the child care staff for autonomous functioning is congruent with the stated aims of how the milieu should run. If, for example, the milieu treatment philosophy of an adolescent ward requires that child care workers serve as models for autonomous and responsible behavior for their adolescent charges, then a leadership structure characterized by strict obedience to the dictates of a Head Nurse would run counter to the milieu's philosophy. Instead of observing self-confident workers who feel that their judgments are valued, in this scheme the adolescent patients would have as models for identification adults who were treated like inept children. Are the lines of authority clear, so that emotionally stressful confusion over decision-making responsibilities does not occur (Henry, 1954)? If not, adolescent patients may once again feel lost as in a family where parenting roles are unclear and limit-setting is deficient or absent. If leadership roles and lines of decision making are not congruent with formal therapeutic goals, understanding their historical development is the first step toward change.

The milieu will contain a variety of informal work roles which spontaneously evolve among the child care workers. They may include: the "nanny," the "policeman," the "kindly uncle," the "sex idol," the "mother," etc. When there is a harmonious fit between role and staff member, morale is boosted and the ward benefits. However, if these informal roles are not continually monitored, discordant person-role fits can occur, resulting in destructive intrastaff and staff-patient tensions. Again, monitoring these roles involves maintaining an understanding of what interaction of people and events led to their development. 
It is likely that each group of care workers harbors myths about the best form of leadership for it. The myth might be a belief that the ward can function only under a matriarchy, with a dominering Head Nurse in charge; or that it can function only under the patriarchal rule of a benevolent but firm male administrator. On the other hand, the dominant belief might be that what is needed is a democracy or a nihilistic disregard for any established principles of authority. Whatever the existing group myth about leadership, it should be recognized and checked against the ward's treatment goals. Particularly if it is not congruent with those goals, the history of such a myth should be discovered. When seen in its historical perspective, a particular myth may be divested of its immutability.

It is also likely that each child care staff harbors myths and traditions about what processes maintain its ward's particular social structure. These may be as general as a need for continual repression of intrastaff feelings or, conversely, an ongoing openness regarding how people feel about the way things are going. However, there may be more specific traditions concerning the cultural mechanisms for periodically renewing the ward's dominant social organization. Such traditions, paralleling the beliefs and religious symbols of larger cultures, may involve an uncounscious belief in the necessity of periodic orgies or outbursts of aggression in order to release pent-up impulses and feelings and, thereby, for a time, to ameliorate socially disruptive forces. Such traditions can directly affect care if child care workers uncounsciously use the patients to enact their myths for them. As with the exploration of other aspects of the milieu's interpersonal structure, understanding the history of such implicit traditions can be the first step toward change.

It should be emphasized that social myths end to reflect far more than simply the staff's defensive distortion of past events. Although we have focused primarily on such distortions, many such myths can accurately capture enduring realities of ward life. These myths can and should be differentiated from those that run contrary to the treatment goals of the ward. Implicit in these positive social myths is the sense of the structure and constancy of the ward community. Especially in the treatment of adolescents who lack an internal sense of structure and identity and whose social world may lack this essential quality of endurance and dependability, it is invaluable for the staff to communicate their own feelings of participation in a community with an enduring identity that reflects a sense of its own history. 


\section{References}

Alt, H. Responsibilities and qualifications of the child care worker. American Journal of Orthopsychiatry, 1953, 23, 670-675.

Bettelheim, B. Training the child care worker in a residential center. American Journal of Orthopsychiatry, 1966, 35, 694-705.

Bettelheim, B., \& Wright, B. Staff development in a treatment institution. American Journal of Orthopsyciatry, 1955, 24, 705-719.

Erikson, E.H. Identity and the Life Cycle: Psychological Issues. New York: International Universities Press, 1959.

Henry, J. The formal structure of a psychiatric hospital. Psychiatry, 1954, 16, 139151.

Henry, J. Types of institutional structure. Psychiatry, 1957, 20, 47-60.

Krohn, A., Miller, D. H., \& Looney, J. Flight from autonomy: Problems of social change in an adolescent inpatient unit. Psychiatry, 1974, 37, 360-371.

Maier, H. W., Hilgeman, I. M., Shuyart, G., \& Loomis, E. A. Supervision of child care workers in a residential treatment service. American Journal of Orthopsychiatry, 1955, 24, 699-704.

Montalvo, B., \& Pavlin, S. Faulty staff communications in a residential treatment center. American Journal of Orthopsychiatry, 1966, 35, 706-711.

Parkes, H. B. Gods and men: The origins of Western culture. New York: Vintage Books, 1965.

Pierce, W. D., Trickett, J., \& Moos, R. Changing ward atmosphere through staff discussion of the perceived ward environment. Archives of General Psychiatry, $1972,26,35-41$.

Rosen, J. S. Personality factors in the reaction of child care workers to emotionally disturbed children. Psychiatry, 1963, 26, 257-265.

Rossman, P., \& Miller, D. H. The effects of social prejudice on hospitilized adolescents. American Journal of Psychiatry, 1973, 130, 1029-1031.

Slater, P. E. Microcosm. New York: Wiley, 1966.

Stanton, A. H., \& Schwartz, M. S. The mental hospital. New York: Basic Books, 1954.

Szurek, S. A. Dynamics of staff interaction in hospital psychiatric treatment of children. American Journal of Orthopsychiatry, 1947, 17, 542-664. 